OPEN ACCESS

Edited by:

James Cheng-Chung Wei, Chung Shan Medical University

Hospital, Taiwan

Reviewed by:

Amita Aggarwal,

Sanjay Gandhi Post Graduate Institute of Medical Sciences (SGPGI), India

David Yu,

University of California, Los Angeles,

United States

*Correspondence:

Mohamed Mandour

m.a.f.m.m.mandour@

amsterdamumc.nl

Specialty section:

This article was submitted to

Autoimmune and

Autoinflammatory Disorders,

a section of the journal

Frontiers in Immunology

Received: 24 April 2021

Accepted: 15 July 2021

Published: 06 September 2021

Citation:

van Tok MN, Mandour M, Wahle J, Labadia ME, van de Sande MGH,

Nabozny G, Baeten DL and

van Duivenvoorde LM (2021)

Paradoxical Augmentation of

Experimental Spondyloarthritis

by RORC Inhibition in HLA-

B27 Transgenic Rats.

Front. Immunol. 12:699987.

doi: 10.3389/fimmu.2021.699987

\section{Paradoxical Augmentation of Experimental Spondyloarthritis by RORC Inhibition in HLA-B27 Transgenic Rats}

Melissa N. van Tok ${ }^{1,2}$, Mohamed Mandour ${ }^{1,2 *}$, Joseph Wahle ${ }^{3}$, Mark E. Labadia $^{3}$, Marleen G. H. van de Sande ${ }^{1,2}$, Gerald Nabozny ${ }^{3}$, Dominique L. Baeten ${ }^{1,2}$ and Leonie M. van Duivenvoorde ${ }^{1,2}$

\footnotetext{
${ }^{1}$ Department of Clinical Immunology and Rheumatology, Amsterdam Rheumatology \& Immunology Center (ARC), Amsterdam University Medical Centers (UMC), Location Academic Medical Center, University of Amsterdam, Amsterdam, Netherlands, ${ }^{2}$ Department of Experimental Immunology, Infection and Immunity Institute, Amsterdam University Medical Centers, Location AMC, University of Amsterdam, Amsterdam, Netherlands, ${ }^{3}$ Immunology and Respiratory Diseases, Boehringer Ingelheim Pharmaceuticals Inc., Ridgefield, CT, United States
}

Objective: IL-17A plays a major role in the pathogenesis of spondyloarthritis (SpA). Here we assessed the impact of inhibition of RAR related orphan receptor- $\gamma(\mathrm{RORC})$, the key transcription factor controlling IL-17 production, on experimental SpA in HLA-B27 transgenic (tg) rats.

Methods: Experimental SpA was induced by immunization of HLA-B27 tg rats with heatinactivated Mycobacterium tuberculosis. Splenocytes obtained at day 7, 14 and 21 after immunization were restimulated ex vivo to assess the induction of pro-inflammatory cytokines. Rats were then prophylactically treated with a RORC inhibitor versus vehicle control. The biologic effect of RORC inhibition was assessed by pro-inflammatory cytokine expression in draining lymph nodes. Arthritis and spondylitis were monitored clinically, and the degree of peripheral and axial inflammation, destruction and new bone formation was confirmed by histology.

Results: Ex vivo mRNA and protein analyses revealed the rapid and selective induction of IL-17A and IL-22 production by a variety of lymphocyte subsets upon disease induction in $\mathrm{HLA}-\mathrm{B} 27$ tg rats. Prophylactic RORC inhibition in vivo suppressed the expression of IL17A, IL17F, and IL-22 without affecting the expression of other $T$ helper cell subset related genes. This biological effect did not translate into clinical efficacy as RORC inhibition significantly accelerated the onset of arthritis and spondylitis, and aggravated the clinical severity of arthritis. This worsening of experimental SpA was confirmed by histopathological demonstration of increased inflammation, destruction, and new bone formation.

Conclusion: Despite a significant suppression of the IL-17 axis, RORC inhibitor treatment accelerates and aggravates experimental SpA in the HLA-B27 tg rat model.

Keywords: spondyloarthritis, IL-17A, RORC, IL-22, HLA-B27 transgenic rats 


\section{INTRODUCTION}

The IL-17 axis plays an important role in the immunopathology of spondyloarthritis $(\mathrm{SpA})$ as indicated by a wealth of genetic, immunopathological, experimental, and translational evidence (1). Randomized clinical trials have formally demonstrated the role of IL-17A, the prototypical IL-17 cytokine, in human SpA. Treatment with Secukinumab (anti-IL-17A mAb) reduced signs and symptoms of ankylosing spondylitis (AS) as well as of psoriatic arthritis (PsA), the major forms of axial and peripheral SpA, respectively $(2,3)$. Similarly, ixekizumab has proven clinical efficacy in PsA (4) and AS (4). Recent evidence, however, suggests that other cytokines of the IL-17A family, with as prime example IL-17F, can contribute to inflammation in SpA (5). Albeit not proven clinically, animal models have also suggested a potential role for IL-22 $(6,7)$. This raises the question whether targeting other molecules in the IL-17 axis could enhance clinical efficacy above and beyond selective IL-17A blockade.

One of the key molecules controlling the production of IL-17A and related cytokines is the transcription factor retinoic acid receptor-related orphan receptor ROR $\gamma t$ (murine) or RORC (the human homologue). RORC is involved in the production and regulation of IL-17A by different cell types including Th17 cells (8), $\gamma \delta \mathrm{T}$ cells (9), innate lymphoid cells (10). In animal models a distinctive group of ROR $\gamma t+$ Tregs that is vital to maintain gastrointestinal homeostasis and avoid colitis (11-13). Moreover, it is crucial in orchestrating the differentiation of naïve CD4 T cells to Th17 cells (8). Enhanced gene transcription of RORC increases IL-17A production in a T cell line and human primary cells (14-16), whereas blockade of RORC in human CD4 $\mathrm{T}$ cells suppresses IL-17A and other inflammatory cytokines, including IL-17F and IL-22 $(17,18)$. Expression of IL-23R, CCR6, and IL-26 were also decreased upon RORC inhibition in Th17 cells, without affecting the gene signature of other $\mathrm{T}$ helper cell types $(18,19)$. Genetic lack of ROR $\gamma t$ protected mice against experimental autoimmune encephalomyelitis (EAE), induced defects in Th17 differentiation and prevented T-cell-transfermediated colitis $(8,20)$. Pre-clinical studies in animal models, including imiquimod-induced psoriasis (21), spontaneous colonic inflammation (22), antigen induced arthritis (17), EAE (23), and experimental autoimmune uveitis (EAU) (24), confirmed that RORC inhibition markedly reduces local and systemic IL-17A levels and decreases tissue inflammation. Moreover, expression of IL-17F and IL-22 was also reduced upon in vivo RORC inhibition $(21,23)$. These findings indicate that RORC could be an interesting therapeutic target in IL-17A dependent pathology, blocking not only IL-17A but also related pro-inflammatory cytokines.

In this study, we aimed to assess RORC as potential therapeutic target in SpA by studying the effects of a small molecule RORC inhibitor in experimental SpA in HLA-B27 transgenic (tg) rats, a well validated model for human SpA (25-27). As we previously showed that both initiation and disease persistence in this model is partially but not completely inhibited by IL-17A blockade (28), we used this model to assess how RORC inhibition affects a panel of IL-17 related cytokines in vivo and if this biological effect translates into clinical efficacy.

\section{METHODS}

\section{Animals}

The inducible HLA-B27 tg $x$ beta-2 microglobulin (B2M) tg rat model has been described previously (27). Briefly, the Tg (HLA$\left.\mathrm{B}^{\star} 2705, \mathrm{~B} 2 \mathrm{M}\right)$ 21-3Reh and $\mathrm{Tg}(\mathrm{B} 2 \mathrm{M})$ 283-2Reh rat lines (25) on Lewis background were bred and housed ( 3 per cage) in individually ventilated cages at the animal research institute AMC. Six weeks-old F1[21-3x283-2] animals were immunized with low dose (30-90 $\mu \mathrm{g}$ depending on sex and housing conditions) heat-inactivated Mycobacterium tuberculosis (MTB) (Difco, Detroit, MI) in $100 \mu \mathrm{l}$ Incomplete Freund's Adjuvant (IFA) (Chondrex) via subcutaneous injection in the tail base. The immunization strategy using heat-inactivated Mycobacterium tuberculosis as a broad innate immune receptor trigger. In the HLA-B27 tg rats, immunization using 30-60 $\mu \mathrm{g}$ M. tuberculosis was sufficient to induce spondylitis and arthritis in both male and female rats (80-100\%). Without immunization spontaneous development of spondylitis and arthritis appeared in $40 \%$ of the male rats around 9 months of age in the presence of a severe autoimmune epididymoorchitis which is clinically manifested in $100 \%$ of the male rats at 3 months of age. Clinical disease (arthritis and spondylitis) appears 20-30 days after immunization. All animal experiments were approved by the AMC Animal Care and Use Committee, in line with national and international regulations and guidelines. The data presented are the data of two separated experiments combined.

\section{Ex Vivo Restimulation Experiments}

Mononuclear cells from spleen were isolated from F1[21-3x283-2] rats at day 7, 14, 21 after immunization with MTB in IFA (n=4/time point). At each time point two non-immunized rats matched by age and gender, were taken along as control. The non-immunized rats $(n=6)$ were pooled for all analyses. Cells were used either unstimulated or after ex vivo restimulation with $10 \mathrm{ng} / \mathrm{ml}$ PMA and $1 \mu \mathrm{g} / \mathrm{ml}$ ionomycin for 6 hours. Using splenocytes, mRNA expression was measured using Taqman assays (Thermo Fisher Scientific) for ill7a (assay ID Rn01757168_m1), thf (assay ID Rn99999017_m1) and ifng (assay ID Rn00594078_m1) with gapdh (assay ID Rn01775763_g1) as housekeeping gene. Data were analyzed according to the $2^{-\Delta \Delta C T}$ method (29). IL-17A, TNF, and IFNy protein secretion was measured by ELISAs in the culture supernatant stimulated for 24 hours (Thermo Fisher Scientific IL-17A ELISA kit \#88-7170-88; R\&D duoset IFNy ELISA \#DY-585). Intracellular protein expression of IL-17A and IFNy (versus isotype control) by different lymphocyte subsets was assessed by FACS in splenocytes stimulated overnight (in the presence of $10 \mu \mathrm{g} / \mathrm{ml}$ Brefeldin for the final 4 hours). Data was recorded using a FACS Canto II and analyzed using FlowJo software. 


\section{In Vivo RORC Inhibition}

The RORC inhibitor BI119 (Boehringer-Ingelheim Pharmaceuticals Inc., Ridgefield, CT, USA) was discovered by screening a small-molecule compound library. BI119 strongly bound to the human RORyt ligand-binding domain (LBD) and was active in an RORyt LBD reporter assay (Kd for RORy LBD $65 \mathrm{nM}$; IC50 for RORy LBD reporter assay $260 \mathrm{nM}$ ). The compound showed high selectivity towards RORyt as demonstrated by a lack of significant activity against RORa $($ IC50 $>10 \mu \mathrm{M})$ and RORb (IC50 > $6 \mu \mathrm{M})$. Rats (n=6/group) received $30 \mathrm{mg} / \mathrm{kg}$ RORC inhibitor dissolved in Natrosol ${ }^{\mathrm{TM}} 250$ Hydroxyethylcellulose (further referred to as Natrosol) (Ashland Specialty Ingredients \#88-7170-88) or Natrosol alone twice daily via oral gavage. Assignment to treatment was random and per cage to avoid cross contamination via feces. Treatment started one week after immunization and continued for five weeks. Experiment was done twice with 6 rats/group.

\section{Serum Exposure Measurement}

Serum samples were collected via Saphenous vein puncture, 2 hours after the morning dose at day 31 and before the morning dose at day 32 post immunization. The concentration of BI114 was determined by liquid chromatography-mass spectrometry analysis.

\section{Downstream Cytokine Analyses}

After in vivo treatment, RNA was isolated from popliteal lymph nodes using TRIzol. Samples were analyzed with a rat Th17 qPCR array according to the manufacturers protocol (Qiagen \# PARN-073Z). Selective genes were confirmed by regular qPCR with SYBR green primers for IL-17A, IL-22, IL-17F, IL-13 and GAPDH as housekeeping gene (all primer sequences are available upon request). Data were analyzed according to the $2^{-\Delta \Delta C T}$ method (29).

\section{Clinical Scoring of Arthritis and Spondylitis}

The presence of arthritis in the paws was determined clinically and digital hind paw swelling was measured with plethysmometry. Arthritis severity in each paw was graded 0-3 as described before (27). Cumulative clinical scores were calculated for severity analysis. Swelling in $\mathrm{cm}^{3}$ was normalized to the days before disease onset. Spondylitis was determined clinically by swelling and bumps in the tail and scored yes/no. In case of humane endpoints, due to ethical considerations, rats were sacrificed with the last observation carried forward. Humane endpoints were defined as $15 \%$ bodyweight loss or two completely swollen paws. One rat in the vehicle treated group was sacrificed due to reaching the humane endpoint for bodyweight loss. Clinical scoring was performed by one observer, blinded for treatment.

\section{Histology}

Hind paws and tails were decalcified in Osteosoft (Merck) and embedded in paraffin. Sections were stained for hematoxylin and eosin or safranin $\mathrm{O}$ and semi-quantitatively scored by two observers blinded for treatment (MT, LD) as previously described (26).

\section{Statistics}

Data were analyzed using GraphPad prism 7 software. Spondylitis and arthritis incidence were analyzed using a survival curve. Comparison of survival curves was analyzed using the Log-Rank (Mantel-Cox) test. Arthritis severity (arthritis score and hind paw swelling) was analyzed using the area under the curve followed by a Mann-Whitney U test. All other data were analyzed using a Mann-Whitney U test.

\section{RESULTS}

\section{Induction of Experimental SpA in HLA-B27 Tg Rats Is Associated With a Rapid and Selective Induction of IL-17A and IL-22}

We previously demonstrated that selective blockade of IL-17A significantly reduces spondylitis and arthritis development in the inducible HLA-B27 tg rat model of SpA (28). To further assess the potential involvement of IL-17A and related pro-inflammatory cytokines, ex vivo cellular responses to MTB immunization were assessed. To this end cytokine expression and production were determined in splenocytes upon restimulation with PMA/ Ionomycin at 7, 14 and 21 days after MTB immunization. RNA expression analysis indicated a trend towards an increase in IL$17 \mathrm{~A}$, but not in IL-17F expression upon restimulation, with the peak-response at 7 days after immunization. Furthermore IL-22 was increased upon restimulation at 7 days after immunization. Other pro-inflammatory cytokines including TNF and IFNy as well as the expression of RORC, were not changed after immunization (Figure 1A). Protein secretion analysis confirmed the trend towards increased levels of IL-17A at 7 days after immunization (Figure 1B), while secretion of TNF and IFNy was not detectable at any time point upon restimulation with PMA/Ionomycin. A repetitive experiment with samples collected at day 7 ( $n=2$ non-immunized and $n=3$ immunized), confirmed the increase in IL-17A expression, and the unchanged expression of IFNy upon immunization. To assess which cells were responsible for IL-17A production in our model FACS analysis was performed, focusing on three T cell subsets: CD4+, CD4- and $\gamma \delta \mathrm{T}$ cells. The increased presence of IL- $17 \mathrm{~A}^{+}$cells at 7 days after immunization could be confirmed, within the population of CD4+ $\mathrm{TCRab}^{+}, \mathrm{CD} 4-\mathrm{TCRab}^{+}$and CD4-TCR $\gamma \delta^{+} \mathrm{T}$ cells (Figure 1C). The frequency of $\mathrm{IFNy}^{+}$cells was low in all subsets, with no differences between the different time points (Figure 1C). Collectively, these data indicate the rapid and selective induction of IL-17A production by a variety $\mathrm{T}$ cell subsets upon MTB immunization in HLA-B27 tg rats.

\section{In Vivo RORC Inhibition Suppresses IL-17A, IL-17F and IL-22 Expression in HLA-B27 Tg Rats}

To assess the potential relevance of RORC - the key transcriptional regulator of IL-17A and related cytokines - as therapeutic target, we performed an in vivo prophylactic treatment study with RORC inhibition versus vehicle control in our HLA-B27 tg at model (Figure 2A). Serum measurements 

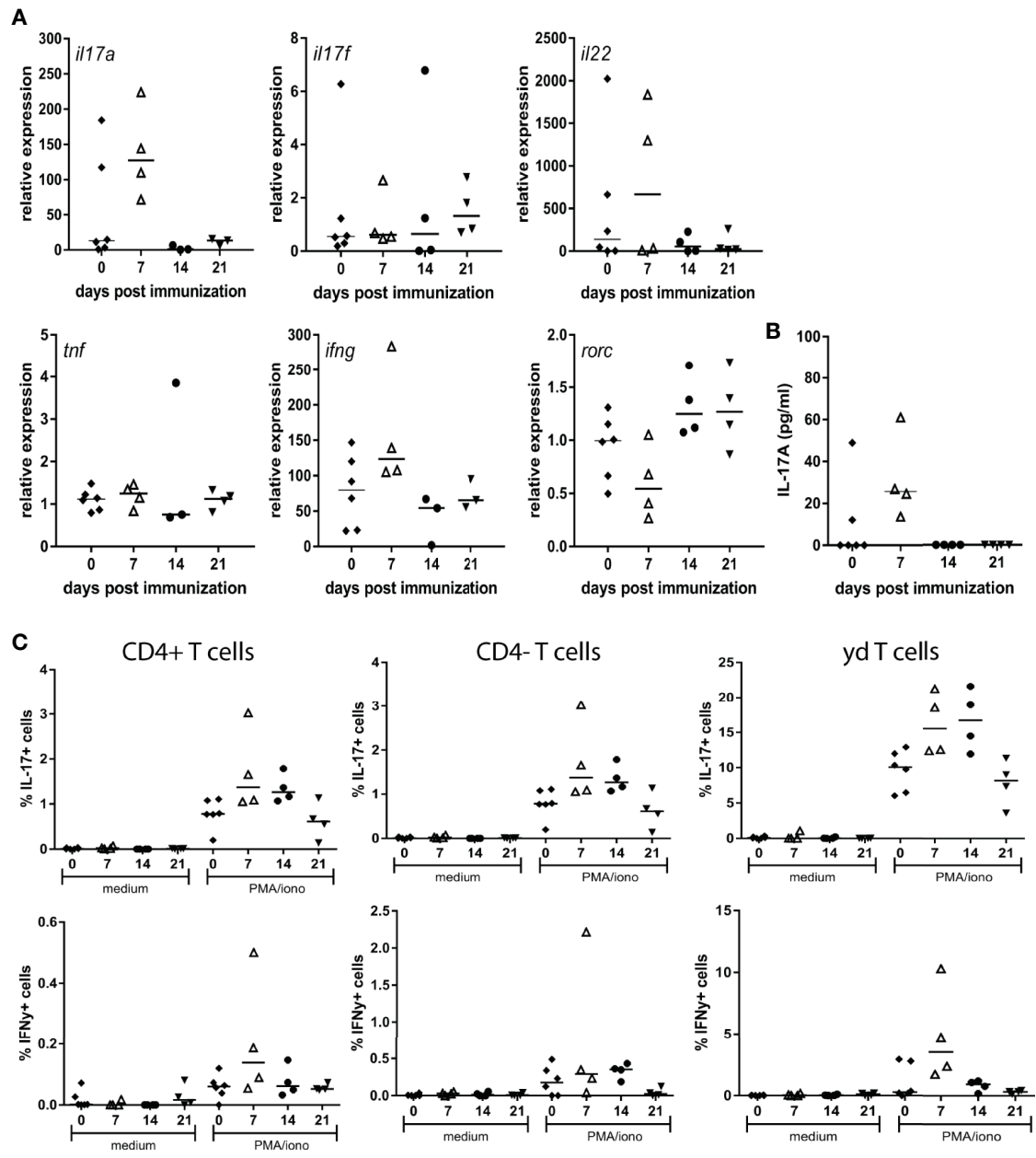

FIGURE 1 | Restimulation of splenocytes from MTB immunized HLA-B27 tg rats primarily induced IL-17A. (A) Relative expression of il17a, il17f, il22, tnf, ifng and rorc (B) protein secretion of IL-17A (C) FACS analysis of IL-17A and IFNy expressing T cell subsets.

confirmed that all rats in the treatment group, but not in the vehicle control group, had high exposure to the compound (Table 1). Whereas a variety of previous experiments with human/mouse cells indicated an IC50 of 10 to $20 \mathrm{nM}$ (data not shown), we confirmed that the exposure levels seen in vivo in the rats significantly inhibited IL-17A production by $\gamma \delta \mathrm{T}$ cells of both wildtype and HLA-B27 tg rats in vitro (Figure 2B).

We next investigated whether the expression of IL-17A and related cytokines was also reduced in vivo by RORC inhibition by analyzing popliteal lymph nodes obtained at the end of the in vivo study. IL-17A, IL-17F, and IL-22 expression was more than 2-fold downregulated as measured in a $\mathrm{GPCR}$ array (Figure 2C). These data were confirmed by regular qPCR (Figure 2D). The Th2 cytokine IL-13 initially showed a tendency towards an increase upon RORC inhibition in the arrays (Figure 2C) but this could not be confirmed by regular qPCR (Figure 2D). Expression of a wide panel of Th1, Th2, and Treg cytokines or transcription factors, or other genes included in the Th17 array, was not different between vehicle treated and RORCi treated rats.
Together these data indicate that RORC inhibition in vivo suppressed the expression of IL-17A, IL17F, and IL-22 without affecting the expression of other $\mathrm{T}$ helper cell subset related genes.

\section{In Vivo RORC Inhibition Augments Experimental SpA in HLA-B27 Tg Rats}

To assess if the observed biological effects on the IL-17 axis translate into clinical efficacy, clinical disease development was monitored over time and histopathological analysis was performed at the end of the study. All RORC inhibitor-treated rats developed both spondylitis and arthritis versus $70 \%$ and $100 \%$, respectively, in the vehicle-treated group (Figure 3A). The mean onset of spondylitis was day 25 in RORC inhibitor versus day 34 in vehicle treated rats. The mean onset of arthritis was day 21 in RORC inhibitor $v s$ day 33 in vehicle treated rats. In terms of arthritis severity, both arthritis score $(\mathrm{p}=0.004)$ and hind paw swelling as assessed by plethysmometry $(p=0.001)$ were increased upon RORC inhibition (Figure 3B). Histopathological analysis of the peripheral joints at the end of the experiment confirmed a 


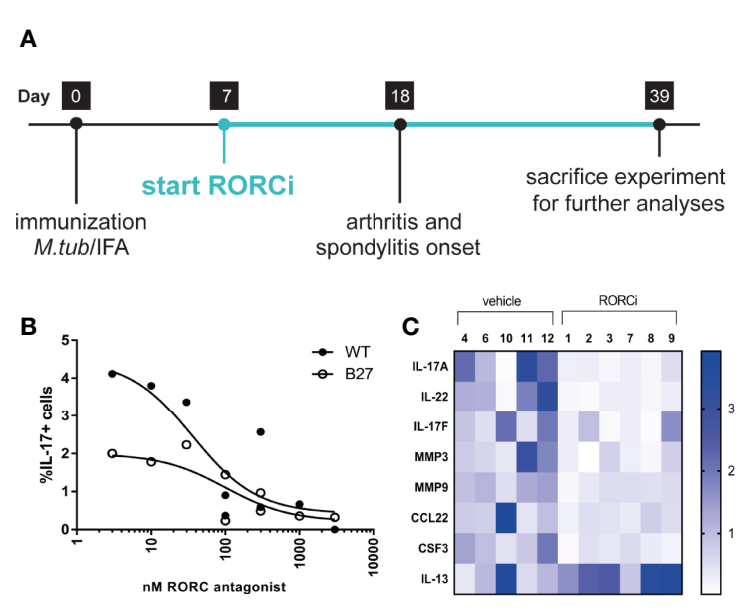

D

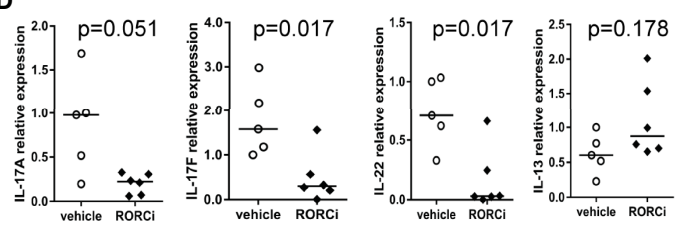

FIGURE 2 | In vivo and in vitro inhibition of RORC. (A) Study design of in vivo prophylactic treatment (B) Dose response effect of in vitro RORC inhibition on frequencies of IL-17+ yd T cells. (C) Relative expression of genes $>2$ fold differentially regulated in vehicle vs RORCi treated rats. (D) Gene expression from the selected genes, IL-17A, IL-22, IL-17F and IL-13 was confirmed by regular $\mathrm{qPCR}$. Data are individual data points and median.

significant increase in inflammatory infiltration $(p=0.004)$, destruction $(p=0.003)$, newly formed bone $(p=0.001)$, and hypertrophic chondrocytes $(\mathrm{p}=0.001)$ in RORC inhibitor treated rats compared to the controls (Figure 4). A similar trend towards increased inflammation $(\mathrm{p}=0.058)$ and bone destruction $(\mathrm{p}=0.061)$ was observed in the spine (Figure 4). Collectively, these data consistently demonstrate that, despite the expected biological impact of RORC inhibition on the IL-17 axis, this treatment did

TABLE 1 | Serum levels of RORC inhibitor.

\begin{tabular}{lccc}
\hline \multirow{2}{*}{ Rat } & Treatment & \multicolumn{2}{c}{ Concentration (nM) } \\
\cline { 3 - 4 } & & before & after \\
\hline 1 & RORCi & 2491 & 2055 \\
2 & RORCi & 1487 & 1576 \\
3 & RORCi & 1425 & 1873 \\
4 & Vehicle & 0 & 0 \\
5 & Vehicle & 0 & 0 \\
6 & Vehicle & 0 & 0 \\
7 & RORCi & 3527 & 4691 \\
8 & RORCi & 1187 & 4673 \\
9 & RORCi & 1891 & 1762 \\
10 & Vehicle & 0 & 0 \\
11 & Vehicle & 0 & 0 \\
12 & Vehicle & 0 & 0 \\
\hline
\end{tabular}

RORCi serum exposure was measured in rats of both groups (RORCi vs vehicle) before the morning dose and 2-3 hours after the morning dose at 1 timepoint only: day 32/33 of treatment. not inhibit but rather accelerated and aggravated experimental SpA in HLA-B27 tg rats. To investigate if RORgt inhibition affected the gut we performed histology on the colon and small intestine. No inflammation was observed in the colon and the small intestine. Combined with the absence of weight loss, these data show that the aggravation of the SpA phenotype cannot be explained by triggering of disease by RORgt inhibition induced gut inflammation.

\section{DISCUSSION}

The major findings of the current study are 1) that IL-17A is primarily produced upon restimulation of splenocytes from MTB immunized rats, with a peak response after 7 days of immunization. 2) IL-17A expression could be reduced upon in vitro as well as in vivo RORC inhibition, additionally in vivo RORC inhibition reduced IL-17F and IL-22 expression without affecting other $\mathrm{T}$ helper cell related genes. 3) Despite these molecular changes RORC inhibition did not inhibit, but rather stimulated disease development. These findings are surprising considering previous studies of RORC inhibition in a variety of inflammatory models including antigen-induced arthritis (17), imiquimod-induced psoriasis (21), IL-23 induced skin inflammation (30), intestinal inflammation $(22,31)$. Recently, Tan et al. (24) showed the reduced clinical severity of experimental autoimmune uveitis (EAU), and EAE using two different RORc antagonists (CQMU151 and CQMU152) (24).

All these models showed decreased levels of IL-17A and significant reduction of clinical symptoms upon pharmacological RORC inhibition or in RORC deficient animals. Also in 2018, Taurog et al. demonstrated the therapeutic efficicacy of the ROR $\gamma t$ antagonist (A-1619758) in reducing inflammation, and suppression of both axial and peripheral skeletal bone changes in the HLA-B27 transgenic rat deficient for Dazl gene (21-3x283-2x17-9) (32).

A major question is whether the data of the current study could have been biased by a technical or biological artefact. Exposure measurements indicated that all rats in the treatment group have high levels of RORC inhibitor present in their serum shortly before and a few hours after treatment. The control group responded as expected, although disease incidence and severity were low. We could detect a biological effect, in terms of reduced IL-17A, IL-17F and IL-22 expression and finally clinical data are in line with histological data. Together these findings prove that groups were not switched during or after this experiment. The potential mechanism remains unknown.

Others showed, using the exact same RORC inhibitor, that while IL-17A levels were reduced, the production of IL-22 continued $(33,34)$ and treatment with the RORC inhibitor abrogated experimental colitis (34). Specific subsets of iNKT and $\gamma \delta$ T cells showed to be among the IL-22 producing cells upon RORC inhibition (33). Similarly another RORC inhibiting compound could selectively impact specific cell types, including Th17 cells, while local IL-17A or IL-22 production by ILC3s was not reduced in a mouse model for intestinal inflammation (22). IL-22 has been shown to be a key player in intestinal host defence and mucosal homeostasis (35), and as we did observe reduced 

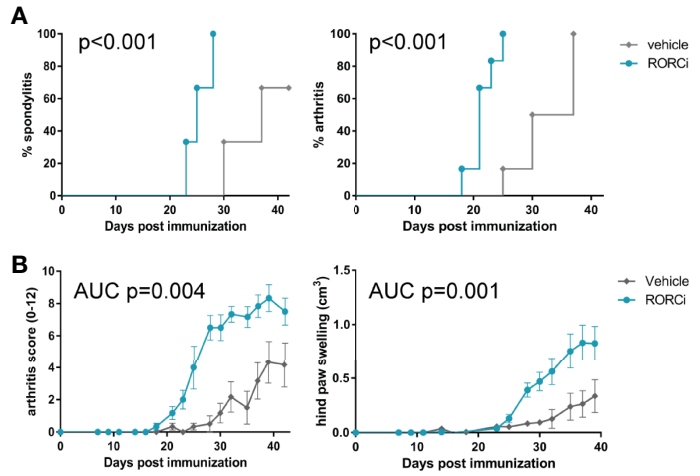

FIGURE 3 | RORC antagonist accelerated spondylitis and arthritis development and increased arthritis severity. (A) Incidence of spondylitis and arthritis in vehicle vs RORCi treated rats (B) disease severity, displayed as arthritis score and hind paw swelling in vehicle vs RORCi treated rats. Data are mean +/- SEM.

IL-22 expression, it could by hypothesized that RORgt inhibition could result in gut inflammation by a decrease in IL-22. This gut inflammation could then induce the SpA phenotype in the rats, similar to the induction of disease by orchitis, which is a consistent finding in this animal model. However, the rats showed no weight loss (Figures 5A, B) and gut inflammation was absent after RORgt inhibition, which makes this hypothesis very unlikely.

In contrast, in another model for intestinal inflammation the RORgt inverse agonist (TAK-828F) suppressed intestinal inflammation. In this model the compound significantly reduced Th17 and Th17/Th1 cell population in mesenteric lymph nodes (MLN) which was accompanied by suppressed/decreased gene expression of not only IL17A, IL17F but also IL-22. Local effects on ILC3 function in the intestine was not studied. However, in normal mice this RORgt inverse agonist did reduce numbers of Th17 cells and IL3s in the laminal propria (31).
In order to test the responsiveness of cells in the MTB immunized HLA-B27 tg rat $\mathrm{CD}^{+} \mathrm{T}$ cells and $\gamma \delta \mathrm{T}$ cells were isolated from spleen and draining lymph nodes and restimulated in the presence of RORC inhibitor ( $n=9$ ). IL-17A and IL-22 expression were similarly decreased in both cell subsets upon RORC inhibition.

It is important to note, however, that this is not the first time that clinical or preclinical data demonstrate that the IL-17 axis does not work in a linear manner. It was reported by Chong et al., that loss of IL-17A produced by Th17 cells didn't reduce the pathogenicity of these cells as expected and instead increased the expression of other Th17 cytokines (i.e. GM-CSF and IL-17F) (36). This was attributed to a Th17 cell-intrinsic autocrine loop induced by IL-17A binding to its receptor resulting in IL-24 induction, which in turn repressed the Th17 cytokine program. They showed that in vivo IL-24 treatment ameliorated Th17-induced EAU, whereas silencing of IL-24 in Th17 cells enhanced disease. However, these findings are unlikely to explain the paradoxical effects of RORC inhibition in our model as we observed the down regulation of other TH17 cytokines (e.g. IL-17F).

Clinical studies in psoriasis patients indicated that both blockade of IL-23 as well as IL-17A are effective $(37,38)$. However in Crohns disease blockade of IL-23 was effective while blockade of IL-17A or IL-17RA was not (39-41). Finally in SpA, blockade of IL-23p19 seems to be effective in psoriatic arthritis (42) but not in ankylosing spondylitis (43). Similarly in a previous study with our HLA-B27 tg rats, we showed that blockade of the IL-23 receptor (IL-23R) significantly reduced levels of IL-17A and IL-22 (but not IL-17F) in vivo. While prophylactic blockade of the IL-23R completely prevented spondylitis and arthritis development, therapeutic blockade did not impact clinical or histological spondyloarthritis in the HLA-B27 tg rat (44). Moreover prophylactic as well as therapeutic blockade of IL-17A was effective in this model (28).

The ROR $\gamma t+$ Tregs subset might be involved in these unanticipated effects. The function of these regulatory cells was thought to be controlled partly by ROR $\gamma t$ (45). Kleinewietfeld et al. showed that a low molecular weight ROR $\gamma$ t inhibitor could influence the frequencies of regulatory T-cells (Tregs) beside the

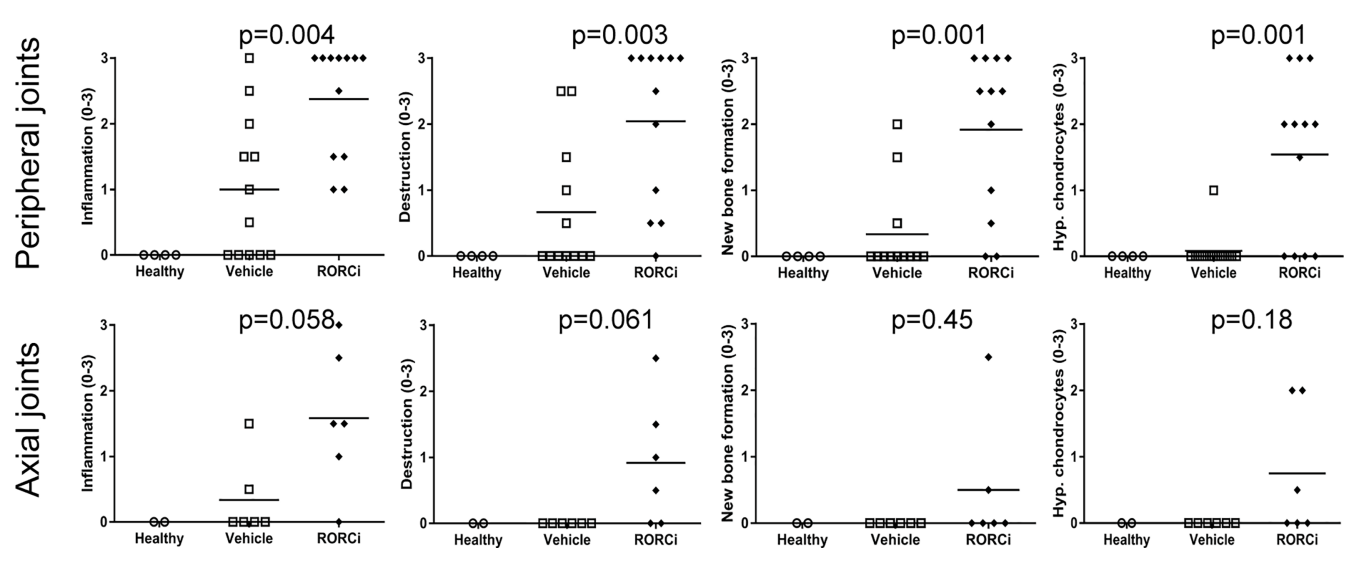

FIGURE 4 | Increased histological pathology in RORC inhibitor treated rats. Peripheral and axial joints were semi-quantitatively scored (0-3) for inflammation, destruction, new bone formation and hypertrophic chondrocytes. 
A

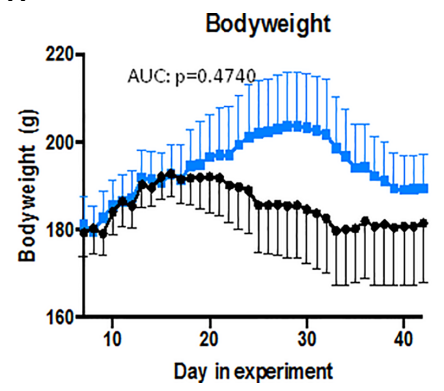

B

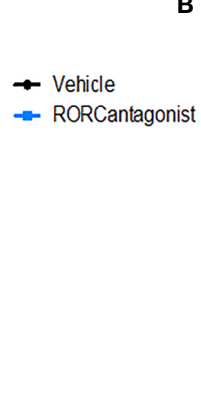

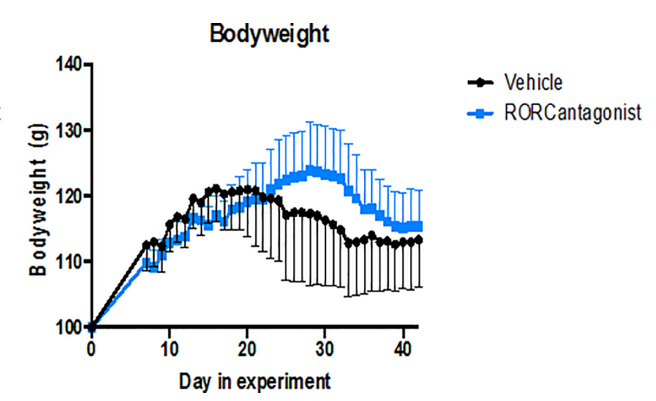

FIGURE 5 | Body weight follow up in treated rats. (A) experiment 1. (B) experiment 2.

Th17 response. The equilibrium between ROR $\gamma t$ and FoxP3 expression level would equally control Treg and Th17 cells, depending on the cytokine environment. ROR $\gamma$ t inhibition would twist the Th17/Treg cell ratio towards the Treg pathway (46). Despite the apparent beneficial effect for inducing immune tolerance, it seems that ROR $\gamma$ t modulation would alter both the downstream pro- and anti-inflammatory pathways and induce undesirable effects (45). Affecting the regulatory function of these RORyt Tregs would predispose to inflammation (even when the expression of Th17 signature genes and cytokines is decreased). The question remains to what extend these findings are relevant for other models or for human disease. Based on our previous findings regarding IL-23R and IL-17A blockade in the HLA-B27 tg rats we conclude that it is uncertain whether RORC inhibition might be a good therapeutic option for all IL-17A driven diseases.

\section{DATA AVAILABILITY STATEMENT}

The original contributions presented in the study are included in the article/supplementary material. Further inquiries can be directed to the corresponding author.

\section{REFERENCES}

1. Yeremenko N, Paramarta JE, Baeten D. The Interleukin-23/Interleukin-17 Immune Axis as a Promising New Target in the Treatment of Spondyloarthritis. Curr Opin Rheumatol (2014) 26(4):361-70. doi: 10.1097/ BOR.0000000000000069

2. Baeten D, Baraliakos X, Braun J, Sieper J, Emery P, van der Heijde D, et al. Anti-Interleukin-17A Monoclonal Antibody Secukinumab in Treatment of Ankylosing Spondylitis: A Randomised, Double-Blind, Placebo-Controlled Trial. Lancet (2013) 382(9906):1705-13. doi: 10.1016/S0140-6736(13)61134-4

3. Baeten D, Sieper J, Braun J, Baraliakos X, Dougados M, Emery P, et al. Secukinumab, An Interleukin-17a Inhibitor, in Ankylosing Spondylitis. N Engl J Med (2015) 373(26):2534-48. doi: 10.1056/NEJMoa1505066

4. Mease PJ, van der Heijde D, Ritchlin CT, Okada M, Cuchacovich RS, Shuler CL, et al. Ixekizumab, An Interleukin-17A Specific Monoclonal Antibody, for T:E Treatment of Biologic-Naive Patients With Active Psoriatic Arthritis: Results From the 24-Week Randomised, Double-Blind, Placebo-Controlled and Active (Adalimumab)-Controlled Period of the Phase III Trial SPIRIT-P1. Ann Rheum Dis (2017) 76(1):79-87. doi: 10.1136/annrheumdis-2016-209709

\section{ETHICS STATEMENT}

All animal experiments were approved by the AMC Animal Care and Use Committee, in line with national and international regulations and guidelines.

\section{AUTHOR CONTRIBUTIONS}

MT and LD, study design, experimental procedures, analyzing data, writing manuscript. MM, analyzing data, writing manuscript. JW, experimental procedures. ML, study design, experimental procedures, analyzing data. MS, analyzing data, writing manuscript. GN and DB, study design, analyzing data, writing manuscript. All authors contributed to the article and approved the submitted version.

\section{FUNDING}

This work was sponsored by Boehringer-Ingelheim Pharmaceuticals Inc., Ridgefield, CT, USA.

5. Glatt S, Baeten D, Baker T, Griffiths M, Ionescu L, Lawson ADG, et al. Dual IL-17A and IL-17F Neutralisation by Bimekizumab in Psoriatic Arthritis: Evidence From Preclinical Experiments and a Randomised PlaceboControlled Clinical Trial That IL-17F Contributes to Human Chronic Tissue Inflammation. Ann Rheum Dis (2018) 77(4):523-32. doi: 10.1136/ annrheumdis-2017-212127

6. Sherlock JP, Joyce-Shaikh B, Turner SP, Chao CC, Sathe M, Grein J, et al. IL-23 Induces Spondyloarthropathy by Acting on ROR-Gammat+ CD3+CD4-CD8Entheseal Resident T Cells. Nat Med (2012) 18(7):1069-76. doi: 10.1038/nm.2817

7. Benham H, Rehaume LM, Hasnain SZ, Velasco J, Baillet AC, Ruutu M, et al. Interleukin-23 Mediates the Intestinal Response to Microbial Beta-1,3-Glucan and the Development of Spondyloarthritis Pathology in SKG Mice. Arthritis Rheumatol (2014) 66(7):1755-67. doi: 10.1002/art.38638

8. Ivanov II, McKenzie BS, Zhou L, Tadokoro CE, Lepelley A, Lafaille JJ, et al. The Orphan Nuclear Receptor RORgammat Directs the Differentiation Program of Proinflammatory IL-17+ T Helper Cells. Cell (2006) 126 (6):1121-33. doi: 10.1016/j.cell.2006.07.035

9. O’Brien RL, Roark CL, Born WK. IL-17-Producing Gammadelta T Cells. Eur J Immunol (2009) 39(3):662-6. doi: 10.1002/eji.200839120 
10. Spits H, Di Santo JP. The Expanding Family of Innate Lymphoid Cells: Regulators and Effectors of Immunity and Tissue Remodeling. Nat Immunol (2011) 12(1):21-7. doi: 10.1038/ni.1962

11. Sefik E, Geva-Zatorsky N, Oh S, Konnikova L, Zemmour D, McGuire AM, et al. MUCOSAL Immunology Individual Intestinal Symbionts Induce a Distinct Population of Rory+ Regulatory T Cells. Science (2015) 349:993-7. doi: $10.1126 /$ science.aaa 9420

12. Ohnmacht C, Park JH, Cording S, Wing JB, Atarashi K, Obata Y, et al. MUCOSAL Immunology the Microbiota Regulates Type 2 Immunity Through Roryt+ T Cells. Science (2015) 349:989-93. doi: 10.1126/ science.aac 4263

13. Yang B-H, Hagemann S, Mamareli P, Lauer U, Hoffmann U, Beckstette M, et al. Foxp3+ T Cells Expressing Roryt Represent a Stabl Regulatory T-Cell Effector Lineage With Enhanced Suppressive Capacity During Intestinal Inflammation. Mucosal Immunol (2015) 205:1381-93. doi: 10.1038/ mi.2015.74

14. Zhang W, Zhang J, Fang L, Zhou L, Wang S, Xiang Z, et al. Increasing Human Th17 Differentiation Through Activation of Orphan Nuclear Receptor Retinoid Acid-Related Orphan Receptor Gamma (RORgamma) by a Class of Aryl Amide Compounds. Mol Pharmacol (2012) 82(4):583-90. doi: 10.1124/mol.112.078667

15. Rene O, Fauber BP, Boenig Gde L, Burton B, Eidenschenk C, Everett C, et al. Minor Structural Change to Tertiary Sulfonamide RORc Ligands Led to Opposite Mechanisms of Action. ACS Med Chem Lett (2015) 6(3):276-81. doi: $10.1021 / \mathrm{ml} 500420 \mathrm{y}$

16. Olsson RI, Xue Y, von Berg S, Aagaard A, McPheat J, Hansson EL, et al. Benzoxazepines Achieve Potent Suppression of IL-17 Release in Human THelper 17 (TH 17) Cells Through an Induced-Fit Binding Mode to the Nuclear Receptor RORgamma. Chem Med Chem (2016) 11(2):207-16. doi: $10.1002 / \mathrm{cmdc} .201500432$

17. Guendisch U, Weiss J, Ecoeur F, Riker JC, Kaupmann K, Kallen J, et al. Pharmacological Inhibition of RORgammat Suppresses the Th17 Pathway and Alleviates Arthritis In Vivo. PloS One (2017) 12(11):e0188391. doi: 10.1371/journal.pone.0188391

18. Guntermann C, Piaia A, Hamel ML, Theil D, Rubic-Schneider T, Del RioEspinola A, et al. Retinoic-Acid-Orphan-Receptor-C Inhibition Suppresses Th17 Cells and Induces Thymic Aberrations. JCI Insight (2017) 2(5):e91127. doi: $10.1172 /$ jci.insight.91127

19. Ecoeur F, Weiss J, Kaupmann K, Hintermann S, Orain D, Guntermann C. Antagonizing Retinoic Acid-Related-Orphan Receptor Gamma Activity Blocks the T Helper 17/Interleukin-17 Pathway Leading to Attenuated ProInflammatory Human Keratinocyte and Skin Responses. Front Immunol (2019) 10:577. doi: 10.3389/fimmu.2019.00577

20. Leppkes M, Becker C, Ivanov II, Hirth S, Wirtz S, Neufert C, et al. RORgamma-Expressing Th17 Cells Induce Murine Chronic Intestinal Inflammation via Redundant Effects of IL-17A and IL-17f. Gastroenterology (2009) 136(1):257-67. doi: 10.1053/j.gastro.2008.10.018

21. Banerjee D, Zhao L, Wu L, Palanichamy A, Ergun A, Peng L, et al. Small Molecule Mediated Inhibition of RORgamma-Dependent Gene Expression and Autoimmune Disease Pathology In Vivo. Immunology (2016) 147(4):399413. doi: $10.1111 / \mathrm{imm} .12570$

22. Withers DR, Hepworth MR, Wang X, Mackley EC, Halford EE, Dutton EE, et al. Transient Inhibition of ROR-Gammat Therapeutically Limits Intestinal Inflammation by Reducing TH17 Cells and Preserving Group 3 Innate Lymphoid Cells. Nat Med (2016) 22(3):319-23. doi: 10.1038/nm.4046

23. Solt LA, Kumar N, Nuhant P, Wang Y, Lauer JL, Liu J, et al. Suppression of TH17 Differentiation and Autoimmunity by a Synthetic ROR Ligand. Nature (2011) 472(7344):491-4. doi: 10.1038/nature10075

24. Tan J, Liu H, Huang M, Li N, Tang S, Meng J, et al. Small Molecules Targeting Roryt Inhibit Autoimmune Disease by Suppressing Th17 Cell Differentiation. Cell Death Dis (2020) 11(8):697. doi: 10.1038/s41419-020-02891-2

25. Tran TM, Dorris ML, Satumtira N, Richardson JA, Hammer RE, Shang J, et al. Additional Human Beta2-Microglobulin Curbs HLA-B27 Misfolding and Promotes Arthritis and Spondylitis Without Colitis in Male HLA-B27Transgenic Rats. Arthritis Rheum (2006) 54(4):1317-27. doi: 10.1002/art.21740

26. van Duivenvoorde LM, Dorris ML, Satumtira N, van Tok MN, Redlich K, Tak $\mathrm{PP}$, et al. Relationship Between Inflammation, Bone Destruction, and Osteoproliferation in the HLA-B27/human Beta2 -Microglobulin-
Transgenic Rat Model of Spondylarthritis. Arthritis Rheum (2012) 64 (10):3210-9. doi: 10.1002/art.34600

27. van Tok MN, Satumtira N, Dorris M, Pots D, Slobodin G, van de Sande MG, et al. Innate Immune Activation Can Trigger Experimental Spondyloarthritis in HLA-B27/Hubeta2m Transgenic Rats. Front Immnol (2017) 8:920. doi: 10.3389/fimmu.2017.00920

28. Van Tok MN, Van Duivenvoorde LM, Kramer I, Ingold P, Pfister S, Roth L, et al. IL-17a Inhibition Diminishes Inflammation And New Bone Formation In Experimental Spondyloarthritis. Arthritis Rheumatol (2019) 71(4):612-25. doi: $10.1002 /$ art.40770

29. Livak KJ, Schmittgen TD. Analysis of Relative Gene Expression Data Using Real-Time Quantitative PCR and the 2(-Delta Delta C(T)) Method. Methods (2001) 25(4):402-8. doi: 10.1006/meth.2001.1262

30. Xue X, Soroosh P, De Leon-Tabaldo A, Luna-Roman R, Sablad M, Rozenkrants N, et al. Pharmacologic Modulation of RORgammat Translates to Efficacy in Preclinical and Translational Models of Psoriasis and Inflammatory Arthritis. Sci Rep (2016) 6:37977. doi: 10.1038/srep37977

31. Igaki K, Nakamura Y, Tanaka M, Mizuno S, Yoshimatsu Y, Komoike Y, et al. Pharmacological Effects of TAK-828F: An Orally Available Roryt Inverse Agonist, in Mouse Colitis Model and Human Blood Cells of Inflammatory Bowel Disease. Inflamm Res (2019) 68(6):493-509. doi: 10.1007/s00011-01901234-y

32. Taurog J, Zhu J, Kort M, Bryant S, Phillips L, Waegell W. Inhibition of the Transcription Factor That Drives IL-17 Expression Suppresses Inflammation, Joint Damage, and New Bone Formation in Experimental Spondyloarthritis in HLA-B27 Transgenic Rats. Abstract Supplement 2018 ACR/ARHP Annual Meeting (Abstract Number 2059). Arthritis Rheumatol (2018) 70(S9):2280-1.

33. Venken 3, Jacques P, Mortier C, Labadia ME, Decruy T, Coudenys J, et al. Roryt Inhibition Selectively Targets IL-17 Producing iNKT and $\gamma \delta$-T Cells Enriched in Spondyloarthritis Patients. Nat Commun (2019) 10(1):9. doi: 10.1038/s41467-018-07911-6

34. Bassolas-Molina H, Raymond E, Labadia M, Wahle J, Ferrer-Picón E, Panzenbeck M, et al. An Roryt Oral Inhibitor Modulates IL-17 Responses in Peripheral Blood and Intestinal Mucosa of Crohn's Disease Patients. Front Immunol (2018) 9:2307. doi: 10.3389/fimmu.2018.02307

35. Sonnenberg GF, Monticelli LA, Alenghat T, Fung TC, Hutnick NA, Kunisawa J, et al. Innate Lymphoid Cells Promote Anatomical Containment of Lymphoidresident Commensal Bacteria. Science (2012) 336:1321-5. doi: $10.1126 /$ science. 1222551

36. Chong WP, Mattapallil MJ, Raychaudhuri K, Bing SJ, Wu S, Zhong Y. The Cytokine IL-17A Limits Th17 Pathogenicity via a Negative Feedback Loop Driven by Autocrine Induction of IL-24. Immunity (2020) 53:384. doi: 10.1016/j.immuni.2020.06.022

37. Papp K, Thaci D, Reich K, Riedl E, Langley RG, Krueger JG, et al. Tildrakizumab (MK-3222), an Anti-Interleukin-23p19 Monoclonal Antibody, Improves Psoriasis in a Phase IIb Randomized PlaceboControlled Trial. Br J Dermatol (2015) 173(4):930-9. doi: 10.1111/bjd.13932

38. Langley RG, Lebwohl M, Krueger GG, Szapary PO, Wasfi Y, Chan D, et al. Long-Term Efficacy and Safety of Ustekinumab, With and Without Dosing Adjustment, in Patients With Moderate-to-Severe Psoriasis: Results From the PHOENIX 2 Study Through 5 Years of Follow-Up. Br J Dermatol (2015) 172 (5):1371-83. doi: 10.1111/bjd.13469

39. Hueber W, Sands BE, Lewitzky S, Vandemeulebroecke M, Reinisch W, Higgins PD, et al. Secukinumab, a Human Anti-IL-17A Monoclonal Antibody, for Moderate to Severe Crohn's Disease: Unexpected Results of a Randomised, Double-Blind Placebo-Controlled Trial. Gut (2012) 61 (12):1693-700. doi: 10.1136/gutjnl-2011-301668

40. Feagan BG, Sandborn WJ, D'Haens G, Panes J, Kaser A, Ferrante M, et al. Induction Therapy With the Selective Interleukin-23 Inhibitor Risankizumab in Patients With Moderate-to-Severe Crohn's Disease: A Randomised, Double-Blind, Placebo-Controlled Phase 2 Study. Lancet (2017) 389 (10080):1699-709. doi: 10.1016/S0140-6736(17)30570-6

41. Sandborn WJ, Feagan BG, Fedorak RN, Scherl E, Fleisher MR, Katz S, et al. A Randomized Trial of Ustekinumab, a Human Interleukin-12/23 Monoclonal Antibody, in Patients With Moderate-to-Severe Crohn's Disease. Gastroenterology (2008) 135(4):1130-41. doi: 10.1053/j.gastro.2008.07.014

42. Mease PJ KH, Morita A, Kivitz AJ, Papp KA, Aslanyan S, Berner B, et al. Efficacy and Safety Results From a Phase 2 Trial of Risankizumab, a Selective 
IL-23p19 Inhibitor, in Patients With Active Psoriatic Arthritis [Abstract]. Arthritis Rheumatol (2017) 69(suppl 10). doi: 10.1136/annrheumdis-2018eular. 2140

43. Baeten D, Ostergaard M, Wei JC, Sieper J, Jarvinen P, Tam LS, et al. Risankizumab, an IL-23 Inhibitor, for Ankylosing Spondylitis: Results of a Randomised, Double-Blind, Placebo-Controlled, Proof-of-Concept, DoseFinding Phase 2 Study. Ann Rheum Dis (2018) 77(9):1295-302. doi: 10.1136/annrheumdis-2018-213328

44. van Tok MN, Na S, Lao CR, Alvi M, Pots D, van de Sande MGH, et al. The Initiation, But Not the Persistence, of Experimental Spondyloarthritis Is Dependent on Interleukin-23 Signaling. Front Immunol (2018) 9:1550. doi: 10.3389/fimmu.2018.01550

45. Mickael ME, Bhaumik S, Basu R. Retinoid-Related Orphan Receptor Roryt in CD4 + T-Cell-Mediated Intestinal Homeostasis and Inflammation. Am J Pathol (2020) 190(10):1984-99. doi: 10.1016/ j.ajpath.2020.07.010

46. Kleinewietfeld M, Hafler DA. The Plasticity of Human Treg and Th17 Cells and Its Role in Autoimmunity. Semin Immunol (2013) 25(4):305-12. doi: 10.1016/j.smim.2013.10.009

Conflict of Interest: ML and GN are employees of Boehringer-Ingelheim Pharmaceuticals Inc. MS received consultancy fees from Novartis and Abbvie and research grants from Janssen, Novartis and Eli Lilly. DB is an employee of UCB.
The authors declare that this study received funding from Boehringer-Ingelheim Pharmaceuticals Inc. The funder shared in study design, data analysis and data collection.

The remaining authors declare that the research was conducted in the absence of any commercial or financial relationships that could be construed as a potential conflict of interest.

Publisher's Note: All claims expressed in this article are solely those of the authors and do not necessarily represent those of their affiliated organizations, or those of the publisher, the editors and the reviewers. Any product that may be evaluated in this article, or claim that may be made by its manufacturer, is not guaranteed or endorsed by the publisher.

Copyright (c) 2021 van Tok, Mandour, Wahle, Labadia, van de Sande, Nabozny, Baeten and van Duivenvoorde. This is an open-access article distributed under the terms of the Creative Commons Attribution License (CC BY). The use, distribution or reproduction in other forums is permitted, provided the original author(s) and the copyright owner(s) are credited and that the original publication in this journal is cited, in accordance with accepted academic practice. No use, distribution or reproduction is permitted which does not comply with these terms. 